\title{
Sturm-Schrödinger equations: formula for metric
}

\author{
Miloslav Znojil \\ Nuclear Physics Institute ASCR, \\ 25068 Řež, \\ Czech Republic* \\ Hendrik B. Geyer \\ Institute of Theoretical Physics, \\ University of Stellenbosch and Stellenbosch Institute for Advanced Study, \\ 7600 Stellenbosch, \\ South Africa ${ }^{\dagger}$
}

November 8, 2018

*e-mail: znojil@ujf.cas.cz

${ }_{\dagger}^{\dagger}$-mail: hbg@sun.ac.za 


\begin{abstract}
Sturm-Schrödinger equations $H \psi=E W \psi$ with $H \neq H^{\dagger}$ and $W \neq W^{\dagger} \neq I$ are considered, with a weak point of the theory lying in the purely numerical matrix-inversion form of the double-series definition of the necessary metric operator $\Theta$ in the physical Hilbert space of states [M. Znojil, J. Phys. A: Math. Theor. 41 (2008) 215304]. This shortcoming is removed here via an amended, single-series definition of $\Theta$.
\end{abstract}

keywords:

cryptohermiticity, Sturm-Schrödinger bound-state problem

PACS:

03.65.Bz, 03.65.Ca 


\section{The origin of Sturm-Schrödinger bound- state equations}

It has already been known to Liouville [1] that after one pays due attention to boundary conditions an elementary change of variables may transform a given Sturm-Liouville eigenvalue problem

$$
H^{(1)} \psi_{n}^{(1)}(r)=E_{n} \psi_{n}^{(1)}(r), \quad H^{(1)}=-\frac{d^{2}}{d r^{2}}+V^{(1)}(r)
$$

into another Sturm-Liouville eigenvalue problem of just slightly more complicated form, viz.,

$$
H^{(2)} \psi_{n}^{(2)}(x)=E_{n} W(x) \psi_{n}^{(2)}(x), \quad H^{(2)}=-\frac{d^{2}}{d x^{2}}+V^{(2)}(x) .
$$

It is important to notice that both these equations are isospectral, i.e., we have $E_{n}^{(1)}=E_{n}^{(2)}=E_{n}$ at all $n=0,1, \ldots$ Recently, we employed such an idea in ref. [2] (to be called paper I in what follows). We interpreted there eq. (10) as a standard Schrödinger equation of Quantum Mechanics, with $H^{(1)}=T+V^{(1)}$ where the differential operator $T$ represented kinetic energy. In parallel, the change of variables (viz., $r \rightarrow x$ and $\psi^{(1)} \rightarrow \psi^{(2)}$ ) has been prescribed in advance so that we arrived at explicit formulae for the two new functions $W(x)$ and $V^{(2)}(x)$ entering the new differential-equation form (2) of our quantum bound-state problem. In such a context we found it very natural to call eq. (2) a Sturm-Schrödinger equation with Hamiltonian $H$ and weight operator $W$.

The principal advantage of our slightly counterintuitive transition from eq. (11) to eq. (2) [notice that we would have $W^{(1)}(r) \equiv 1$ in eq. (1)] has been found in a perceivable simplification of the physical asymptotic boundary conditions. This implied that also the practical (say, numerical) solution of 
eq. (2) proved much easier and quicker than the solution of its predecessor (11) (cf. refs. 2] or [3] for more details).

\section{The emergence of a nontrivial metric in Sturm-Schrödinger bound-state problems in Quantum Mechanics}

One must be aware that the presence of the nontrivial weight operator $W(x)$ may make the solution of eq. (2) less routine. In paper I we paid particular attention to models (11) and (2) where in spite of the reality of all the spectrum of energies a naive choice of the two alternative representations $\mathcal{H}_{(1,2)}^{(F)}$ of the Hilbert space of states of our quantum system in question happened to lead to inconsistencies like negative probabilities etc. For this reason the superscript $(F)$ stands here for "false".

In paper I we emphasized that the superscript ${ }^{(F)}$ could equally well stand here for "friendly". Indeed, once we temporarily forget about inner products and consider just the underlying two vector spaces $\mathcal{V}_{(1,2)}^{(F)}$ we may treat them simply as spanned by the respective eigenfunctions or generalized eigenfunctions $\psi_{n}^{(1,2)}(\cdot) \equiv\left\langle\cdot \mid \psi_{n}^{(1,2)}\right\rangle$ of our respective Hamiltonians.

In such a setting the main difficulty as addressed in paper I was represented by our specific assumption of the manifest non-Hermiticity of our Hamiltonian operators,

$$
H^{(1,2)} \neq\left[H^{(1,2)}\right]^{\dagger} \quad \text { in } \quad \mathcal{H}^{(F)}=\mathcal{H}_{(1,2)}^{(F)} .
$$

Fortunately, being well aware of the inadequacy of the underlying choice of the specific and most elementary (often called Dirac's or Lebesgue's) inner 
products

$$
\langle\psi \mid \phi\rangle=\int d \mu(r) \psi^{*}(r) \phi(r), \quad|\psi\rangle,|\phi\rangle \in \mathcal{H}^{(F)}
$$

we immediately recalled the well known recipe as described, e.g., in refs. [4] or [5].

Let us briefly recollect the mathematical core of the recipe which lies in a redefinition of the operation of Hermitian conjugation. Technically this means that in the first step we decide to treat our Hilbert space $\mathcal{H}^{(F)}$, formally, as a vector space $\mathcal{V}^{(F)}$ of kets $|\psi\rangle$ complemented by the "usual" definition of the dual space $\left[\mathcal{V}^{(F)}\right]^{\prime}$ (marked by a prime) of bras $\langle\psi|$. In this setting the operation of Hermitian conjugation $\mathcal{T}^{(\text {general) }}$ is, in general, non-unique [6]. Still, people usually ignore this ambiguity and decide to work in full analogy with the linear algebra and finite-dimensional Hilbert spaces. Thus, they specify the dual vector space of linear functionals, i.e., bra vectors as quantities constructed from their ket-vector partners by the very specific Dirac's operation $\mathcal{T}^{\text {(Dirac) }}$ of transposition plus complex conjugation.

In the second step we replace the above-mentioned Dirac's definition of conjugation in Hilbert space $\mathcal{H}^{(F)}$, viz.,

$$
\left\langle\psi\left|:=\mathcal{T}^{(\text {Dirac })}\right| \psi\right\rangle \quad \text { in } \quad \mathcal{H}^{(F)}
$$

by a non-Dirac definition of conjugation which amounts to an introduction of another Hilbert space $\mathcal{H}^{(S)}$. This new, unitarily non-equivalent (i.e., inner product non-preserving) representation of Hilbert space is to be formed by the same vector space $\mathcal{V}^{(S)} \equiv \mathcal{V}^{(F)}$ and by a different "primed" vector space of linear functionals $\left[\mathcal{V}^{(S)}\right]^{\prime} \neq\left[\mathcal{V}^{(F)}\right]^{\prime}$.

On the level of notation we must graphically distinguish between the dual elements $\langle\psi| \in\left[\mathcal{V}^{(F)}\right]^{\prime}$ given by eq. (5) and, in our notation [2], elements 
$\left\langle\langle\psi| \in\left[\mathcal{V}^{(S)}\right]^{\prime}\right.$ defined by the different relation

$$
\left\langle\langle\psi| \equiv\left\langle\psi\left|\Theta:=\mathcal{T}^{(\text {non-Dirac })}\right| \psi\right\rangle \quad \text { in } \quad \mathcal{H}^{(S)}, \quad \Theta \neq I\right.
$$

or, in the coordinate representation,

$$
\left\langle\langle\psi \mid \cdot\rangle=\int d \mu(r) \psi^{*}(r) \Theta(r, \cdot) .\right.
$$

This redefinition realizes the transition from the false Hilbert spaces $\mathcal{H}_{(1,2)}^{(F)}$

to their unitarily non-equivalent alternatives $\mathcal{H}_{(1,2)}^{(S)}$ where the superscript ${ }^{(S)}$ stands for "standard". In the coordinate represenation one speaks about the modified, double-integral definition of the inner product

$$
\left\langle\langle\psi \mid \phi\rangle=\int d \mu(x) \int d \mu(y) \psi^{*}(x) \Theta(x, y) \phi(y)\right.
$$

applicable to any two wave functions $\psi$ and $\phi$ in $\mathcal{H}_{(1,2)}^{(S)}$.

\section{$3 \quad$ Generalized Dyson-type mappings $\Omega$}

The key purpose of introducing the above-described nontrivial metrics $\Theta=$ $\Theta_{(1,2)}$ lies in making our Hamiltonians self-adjoint in $\mathcal{H}_{(1,2)}^{(S)}$. For our further purposes we shall drop the subscripts and restrict our attention just to the Sturmian cases with subscripts ${ }_{2}$. Next we introduce an invertible map $\Omega$ : $\mathcal{V} \rightarrow \mathcal{A}$ connecting our vector space of functions (with ket elements $|\phi\rangle \in \mathcal{V}$ ) with another, abstract vector space $\mathcal{A}$ composed of certain not yet specified spiked-ket elements,

$$
|\psi \succ=\Omega| \psi\rangle, \quad|\psi\rangle \in \mathcal{V}, \quad \mid \psi \succ \in \mathcal{A} .
$$

Using such a notation we shall assume the solvability of the non-selfadjoint doublet of the Sturm-Schrödinger equations composed of eq. (2) and of its dual 
defined in the sense of space $\mathcal{H}^{(F)}$, i.e., in the same vector space $\mathcal{V}$. Thus, in an obvious shorthand notation we shall consider the following two equations,

$$
\left.\left.H|\lambda\rangle=\lambda W|\lambda\rangle, \quad H^{\dagger}\left|\lambda^{\prime}\right\rangle\right\rangle=\lambda^{\prime} W^{\dagger}\left|\lambda^{\prime}\right\rangle\right\rangle
$$

By assumption, they define two families of elements of vector space $\mathcal{V}$. After the application of mapping $\Omega$ one also obtains the corresponding families of elements of the abstract vector space $\mathcal{A}$. Working here, exclusively and solely, with the standard and usual Dirac's conjugation we may introduce also the dual space $\mathcal{A}^{\prime}$ and a related third, abstract Hilbert space $\mathcal{H}^{(P)}$ where the superscript $(P)$ stands for "physical".

These conventions lead to several immediate consequences. Firstly, without any danger of confusion, the elements of the dual space of linear functionals may simply be denoted by the spiked ket symbols,

$$
\prec \psi \mid=\langle\psi| \Omega^{\dagger} \in \mathcal{A}^{\prime} .
$$

Secondly, the lower-case isospectral equivalent $h=\Omega H \Omega^{-1}$ of our original non-Hermitian upper-case Hamiltonians $H \neq H^{\dagger}$ as well as the parallel partner $w=\Omega W \Omega^{-1}$ of any original non-Hermitian specific "weight" operator $W \neq$ $W^{\dagger}$ may and will be assumed self-adjoint in the corresponding abstract physical Hilbert space $\mathcal{H}^{(P)}$. In a way explained in ref. [5] the latter space is specified by its spiked-ket elements (8) and by its spiked-bra linear functionals (10).

All this means that we must require that

$$
h^{\dagger}=\left(\Omega^{-1}\right)^{\dagger} H^{\dagger} \Omega^{\dagger}=h, \quad w^{\dagger}=\left(\Omega^{-1}\right)^{\dagger} W^{\dagger} \Omega^{\dagger}=w,
$$

or, after a trivial re-arrangement,

$$
H^{\dagger}=\Theta H \Theta^{-1}, \quad W^{\dagger}=\Theta W \Theta^{-1}
$$


where we abbreviated $\Theta=\Omega^{\dagger} \Omega$. This shows how the concept of Hermiticity in physical Hilbert space $\mathcal{H}^{(P)}$ remains equivalent to the apparent non- or pseudo-Hermiticity or cryptohermiticity in $\mathcal{H}^{(F)}$. In the language of ref. [4], property (11) of all our operators should be called quasi-Hermiticity in space $\mathcal{H}^{(S)}$ where the metric is nontrivial.

The pull-back of all the theory to the abstract Hilbert space $\mathcal{H}^{(P)}$ clarifies that all our present considerations need not leave the domain covered by standard textbooks [6]. In particular, due to the assumption of invertibility of the mappings $\Omega$ we may replace our two original upper-case Sturmian problems (9) by their common lower-case reincarnation

$$
h|\lambda \succ=\lambda w| \lambda \succ
$$

which is necessarily self-adjoint in $\mathcal{H}^{(P)}$. Its simplicity facilitates the derivation of the Sturmian orthogonality relations

$$
\prec \lambda|w| \lambda^{\prime} \succ=\prec \lambda|w| \lambda \succ \quad \delta_{\lambda, \lambda^{\prime}}
$$

and of the Sturmian completeness relations,

$$
I=\sum_{\lambda}\left|\lambda \succ \frac{1}{\prec \lambda|w| \lambda \succ} \prec \lambda\right| w
$$

as well as of the Sturmian spectral representation

$$
h=\sum_{\lambda} w\left|\lambda \succ \frac{\lambda}{\prec \lambda|w| \lambda \succ} \prec \lambda\right| w
$$

of the lower-case Hamiltonian in $\mathcal{H}^{(P)}$. 


\section{Biorthogonal bases and the double-series formula for the metric}

Our original Hilbert space $\mathcal{H}^{(F)}$ is, by assumption, so simple that it makes sense to transfer all the above formulae to this space. Thus, the insertion of definitions (8) and (10) in eq. (13) yields the orthogonality relations in $\mathcal{H}^{(F)}$,

$$
\left\langle\lambda\left|\Omega^{\dagger} w \Omega\right| \lambda^{\prime}\right\rangle=\left\langle\lambda|\Theta W| \lambda^{\prime}\right\rangle=\langle\lambda|\Theta W| \lambda\rangle \cdot \delta_{\lambda, \lambda^{\prime}}
$$

Similarly, the appropriately adapted version of the completeness is obtained,

$$
I=\sum_{\lambda}|\lambda\rangle \frac{1}{\langle\lambda|\Theta W| \lambda\rangle}\langle\lambda| \Theta W
$$

Finally, the spectral decomposition of the Hamiltonian acquires the following form,

$$
H=\sum_{\lambda} W|\lambda\rangle \frac{\lambda}{\langle\lambda|\Theta W| \lambda\rangle}\langle\lambda| \Theta W .
$$

Moreover, the double-ket eigenstates $|\lambda\rangle\rangle$ of $H^{\dagger}$ may be understood as equal to the products $\Theta|\lambda\rangle[2,5]$.

The key benefit of our return to the metric-independent and computationfriendly space $\mathcal{H}^{(F)}$ is that we may evaluate and set the matrix elements $\langle\lambda|\Theta W| \lambda\rangle$ in eq. (16) equal to one via a suitable normalization of the basis [5]. This convention will perceivably simplify our formulae. The simplification would remain applicable whenever the Hermitian product $\Theta W$ stays positive definite (which is to be assumed). The first consequence of the resulting simplification of the formulae is that we may introduce the further, curly-ketmarked wave-function vectors and their curly-ketket partner vectors defined, respectively, as follows,

$$
\left.\mid \psi\}=W|\psi\rangle, \quad \mid \psi\}\}=W^{\dagger}|\psi\rangle\right\rangle
$$


In terms of these new abbreviations we may stay working inside the most comfortable Hilbert space $\mathcal{H}^{(F)}$ and write down the following alternative and optional forms of the orthogonality conditions,

$$
\left\langle\lambda|\Theta W| \lambda^{\prime}\right\rangle=\left\{\left\{\lambda\left|\lambda^{\prime}\right\rangle=\left\langle\langle\lambda| \lambda^{\prime}\right\}=\delta_{\lambda, \lambda^{\prime}} .\right.\right.
$$

Similarly we may derive the two alternative forms of the completeness relations,

$$
I=\sum_{\lambda}|\lambda\rangle\left\{\left\{\lambda\left|=\sum_{\lambda}\right| \lambda\right\}\langle\langle\lambda|\right.
$$

Finally, the menu of several alternative spectral-representation expansions can be replaced by the more or less unique, most compact expressions

$$
\left.W=\sum_{\lambda} \mid \lambda\right\}\left\{\left\{\lambda\left|, \quad H=\sum_{\lambda}\right| \lambda\right\} \lambda\{\{\lambda \mid\right.
$$

representing the weights and the Hamiltonian operators.

At this moment one could decide to employ the method of ref. [2] and to insert spectral formulae (21) in the fundamental quasi-Hermiticity constraint or equation $H^{\dagger} \Theta=\Theta H$ yielding

$$
\left.\left.\sum_{\lambda} \mid \lambda\right\}\right\} \lambda\left\{\lambda\left|\Theta=\sum_{\lambda} \Theta\right| \lambda\right\} \lambda\{\{\lambda \mid
$$

This relation would strongly suggest that the most natural Sturmian analogue of the well known single-series $W=I$ formula should be sought via the double series ansatz

$$
\left.\left.\Theta=\sum_{\lambda, \lambda^{\prime}} \mid \lambda\right\}\right\} M_{\lambda, \lambda^{\prime}}\left\{\left\{\lambda^{\prime} \mid, \quad M_{\lambda, \lambda^{\prime}}=\left\langle\lambda|\Theta| \lambda^{\prime}\right\rangle .\right.\right.
$$

This approach has been accepted in paper I and contributed to its discouraging conclusions. Fortunately, a better strategy exists. Its core and main consequences will now be described in our last section 5. 


\section{The single-series formula for $\Theta=\Theta(H, W)$}

We arrived at the heart of our present message. The identity $|\psi\rangle\rangle=\Theta|\psi\rangle$ inspires us to start from eq. (20) and to obtain the following unexpected but sufficiently simple result

$$
\left.\Theta=\sum_{\lambda}|\lambda\rangle\right\rangle\{\{\lambda \mid
$$

The impression of an apparent non-Hermiticity of this asymmetric formula is

misleading and it is virtually trivial to verify that $\Theta=\Theta^{\dagger}$ as required.

We should add that as long as the less economical, double-series formula (23) for the metric is concerned, it might still find some marginal applications in some more complicated quantum toboggans [7] etc. Moreover, its use may always be combined with our present, single-series recipe. For example, in comparison with the recommendations formulated in ref. [2], the necessary nondiagonal matrix coefficients are now much more easily evaluated,

$$
M_{\lambda, \lambda^{\prime}}=\left\langle\lambda|\Theta| \lambda^{\prime}\right\rangle=\left\langle\left\langle\lambda \mid \lambda^{\prime}\right\rangle=\left\{\left\{\lambda\left|W^{-1}\right| \lambda^{\prime}\right\rangle=\left\langle\left\langle\lambda\left|W^{-1}\right| \lambda^{\prime}\right\} .\right.\right.\right.
$$

Moreover, the single-series expansion (24) of the Sturmian metric could prove too compact when the analysis of some symmetries is concerned.

Of course, the advantages of our present single-series formula will prevail in the majority of applications where, typically, people truncate the infinite series in order to obtain a reasonable approximation of observable quantities [8], etc. Moreover, even without immediate numerical applications, the existence and compact form of such a formula can definitely be seen as an important indication of the mathematical as well as physical consistency and tractability of the whole family of non-Hermitian, cryptohermitian Sturmian models. 


\section{Summary}

The standard areas of applicability of the general concept of Sturmians has been reviewed by Rotenberg [9]. The scope of this review ranges from the electron-hydrogen scattering to the interatomic charge-transfer collisions and to the atomic-physics related solutions of the Faddeev and Born-Oppenheimer dynamical and phenomenological equations. In the not too remote area of mathematical physics the transition from bound states [cf. eq. (1)] to Sturmians [cf. eq. (22)] found typical applications in perturbation theory [10].

Besides such a role aiming at an immediate determination of energy-dependent couplings the phenomenological use of Sturmians involves mathematically motivated constructions of the so called quasi-exactly solvable quantum models [11. The latter models with $W(r) \sim r^{M}$ at positive (half)integer $M$ re-attracted attention to many open questions like, e.g., the completeness of the sets of the Sturmian wave functions [12. Last but not least, one should keep in mind that certain exact Sturmians emerging at $W(r)=r^{N}$ with any $N=-1,0,1,2, \ldots$ proved helpful, in the context of classical physics, in connection with the resonant internal boundary layer problem [13].

Our preceding paper I revisited the problem of Sturmians and extended the domain of their applicability to the models characterized by a loss of manifest Hermiticity of the Hamiltonian. It has been established there that there exists a close connection between the concepts of Sturmians and of the so called crypto-Hermitian Hamiltonians with real spectra. We emphasized there that the survival of the reality of the energy spectrum can be expected to occur not only in the non-Sturmian regime with $W=I$ but also in the presence of the nontrivial weight operators $W \neq I$.

In our present continuation of paper I we felt inspired by the lasting interest 
in the applications of Sturmians mediated by the problems ranging from the very standard computational physics [14] to the rather exotic tobogganic models using a fairly abstract concept of complexified coordinates [2, 7]. In this setting we perceive paper I as an introduction to the subject which, naturally, suffered from an incomplete understanding of many subtleties.

One of these subtleties has been clarified in our present paper. Our main conclusion is that the parallels between Schrödinger equations of the respective types (11) and (21) are much closer than expected in paper I. In particular, we feel pleased by the fact that in contrast to the very sceptical expectations expressed in paper I we succeeded here in finding the entirely general formula for the metric $\Theta$ and in showing that this operator of key relevance can be expressed as a single infinite sum over certain elementary projectors at $W=I$ as well as at $W \neq I$, i.e., in both the non-Sturmian and Sturmian cases, respectively.

\section{Acknowledgement}

Participation of MZ supported by the MŠMT "Doppler Institute" project Nr. LC06002, by GAČR, grant Nr. 202/07/1307 and, last but not least, by the hospitality of NiTheP and STIAS in Stellenbosch. 


\section{References}

[1] L. Liouville, J. Math. Pures Appl. 1 (1837) 16;

M. Znojil, J. Phys. A: Math. Gen. 27 (1994) 4945.

[2] M. Znojil, J. Phys. A: Math. Theor., to appear (arXiv:0803.0403).

[3] G. J. C. Wessels, A numerical and analytical investigation into nonHermitian Hamiltonians (Master-degree thesis supervised by H. B. Geyer and J. A. C. Weideman, University of Stellenbosch, 2008).

[4] F. G. Scholtz, H. B. Geyer and F. J. W. Hahne, Ann. Phys. (NY) 213 (1992) 74 .

[5] M. Znojil, SIGMA 4 (2008) 001, arXiv overlay: 0710.4432v3 [math-ph].

[6] A. Messiah, Quantum Mechanics I, North Holland, Amsterdam, 1961.

[7] M. Znojil, Phys. Lett. A 372 (2008) 584 and 3591.

[8] A. Mostafazadeh and A. Batal, J. Phys. A: Math. Gen. 37 (2004) 11645;

A. Mostafazadeh, J. Phys. A: Math. Gen. 38 (2005) 6557.

[9] M. Rotenberg, Adv. At. Mol. Phys. 6 (1970) 233.

[10] M. Znojil, J. Math. Phys. 38 (1997) 5087.

[11] A. Voros, J. Phys. A: Math. Gen. 33 (2000) 7423.

[12] R. Szmytkowski and B. Zywicka-Mozejko, Phys. Rev. A 62 (2000) 022104.

[13] C. M. Bender and Q. Wang, J. Phys. A: Math. Gen. 34 (2001) 9835.

[14] E. Kelbert, A. Hyder, F. Demir, Z. T. Hlousek and Z. Papp, J. Phys. A: Math. Theor. 40 (2007) 7721. 